\title{
Outbreak of Foodborne Botulism Associated with Improperly Jarred Pesto - Ohio and California, 2014
}

\author{
Patrick Burke, $\mathrm{MPH}^{1}$; Michael Needham, $\mathrm{MPH}^{2}$; Brendan R. Jackson, $\mathrm{MD}^{3}$; Rick Bokanyi, $\mathrm{PhD}^{4}$; Eric St. Germain ${ }^{4}$; Steven J. Englender, MD ${ }^{1}$
}

On July 28, 2014, the Cincinnati Health Department was notified of suspected cases of foodborne botulism in two women admitted to the same hospital 12 days apart. Patient A had been treated for 12 days for suspected autoimmune disease. When patient $B$, the roommate of patient $A$, was evaluated at the same medical center for similar symptoms, it was learned that on July 13, patient A and patient B had shared a meal that included prepackaged pesto from a jar; clinicians suspected botulism and notified the local health department. The pesto had been purchased from company A's farm stand in San Clemente, California. Laboratory testing detected botulinum toxin type $\mathrm{B}$ by enzyme-linked immunosorbent assay (ELISA) in leftovers of pasta with pesto. A culture of these food samples yielded Clostridium spp. that produced botulinum toxin type B; polymerase chain reaction (PCR) testing also was positive for type B toxin gene. Environmental assessment of company A identified improper acidification and pressurization practices and lack of licensure to sell canned products commercially, including products in hermetically-sealed jars. On July 30, the vendor voluntarily recalled all jarred products, and the California Department of Public Health (CDPH) warned the public not to consume company A's jarred foods. This report describes the two cases and the public health investigation that traced the source of the outbreak.

\section{Patient A}

On the evening of July 15, 2014, patient A, an otherwise healthy woman aged 20 years, was evaluated at the emergency department (ED) of hospital A, reporting 12 hours of worsening throat pain. She received a diagnosis of pharyngitis and was discharged with a prescription for antibiotics. The next day she returned to the same ED with worsening symptoms, including inability to swallow, double vision on lateral gaze, and decreased sensation in her right arm. She was admitted because of concern for airway compromise. She was noted to have dysarthria and nasal speech; however, a motor examination of the arms and legs was normal. A neurologist described these clinical findings as consistent with myasthenia gravis or Miller Fisher syndrome, a rare, acquired nerve disease that is considered to be a variant of Guillain-Barré syndrome. Neostigmine challenge and acetylcholine receptor antibody test were not consistent with myasthenia gravis. Cerebrospinal fluid analyses and magnetic resonance imaging of the brain were unremarkable.
On July 19, patient A was transferred to the neurologic intensive care unit (ICU) of facility B with worsening bulbar symptoms, where she was intubated for impending respiratory failure and treated with 5 days of intravenous immunoglobulin. On July 27, a physician suspected botulism as the likely diagnosis after learning that patient $\mathrm{A}$ had shared a meal with patient $\mathrm{B}$, who had recently been admitted for neurologic dysfunction. A July 30 nerve conduction study and electromyogram demonstrated a presynaptic defect in neuromuscular junction function, suggestive of botulism. Patient A was not treated with botulinum antitoxin because 17 days had elapsed since exposure and there was evidence of clinical improvement. She was transferred to a long-term acute care facility on August 1 and discharged home 22 days later.

\section{Patient B}

On the evening of July 16, 2014, patient B, an otherwise healthy woman aged 22 years, was evaluated at facility A's ED for a sore throat. She made three additional ED visits to a different health care facility (facility C) on July 18, 19, and 23, reporting difficult and painful swallowing, nausea, abdominal pain, and dehydration. Over the course of these visits, she received a prescription for amoxicillin, an injection of penicillin, and oral corticosteroids for presumed tonsillitis. On July 27, patient B went to the ED of facility B with difficulty speaking, progressive weakness, and shortness of breath. Later that day, she developed upper extremity weakness, ptosis, diplopia, and hoarse voice and was admitted to the neurologic ICU and intubated. Botulism was suspected after the link to patient $A$ was identified, and botulinum antitoxin was administered to patient B on the evening of July 28. Patient B was transferred to a long-term acute care facility on August 6, and discharged home 9 days later.

Clinical specimens from the two patients were sent to the Ohio Department of Health Laboratory (ODHL) for Clostridium botulinum testing by culture and mouse bioassay. All clinical specimens were collected $\geq 12$ days after the shared meal, and tests were negative (Table).

\section{Public Health Investigation}

After being notified of the possible botulism cases, Cincinnati Health Department epidemiologists interviewed the two patients and their families. The two patients reported sharing a meal of baked chicken breasts, boiled pasta, steamed 
Morbidity and Mortality Weekly Report

TABLE. Results of laboratory testing for botulinum toxin-producing Clostridium spp. and botulinum toxin type B in clinical specimens and food samples collected during an outbreak investigation - Ohio, Colorado, and California, July-August 2014

\begin{tabular}{|c|c|c|c|c|c|}
\hline Specimen/Sample & Date collected & ELISA* & $\mathrm{PCR}^{\dagger}$ & Mouse bioassay* & Culture ${ }^{\S}$ \\
\hline \multicolumn{6}{|l|}{ Patient specimen } \\
\hline Serum (patient A) & July 25 & NP & NP & Negative & NP \\
\hline Serum (patient A) & July 28 & NP & NP & Negative & NP \\
\hline Serum (patient B) & July 28 & NP & NP & Negative & NP \\
\hline Stool (patient A) & July 25 & NP & NP & Negative & Negative \\
\hline Stool (patient A) & August 4 & NP & NP & Negative & Negative \\
\hline Stool (patient B) & July 29 & NP & NP & Negative & Negative \\
\hline Stool (patient B) & August 5 & NP & NP & Negative & Negative \\
\hline \multicolumn{6}{|l|}{ Food sample } \\
\hline Pasta with pesto 1 & July 28 & Positive & Positive & Inconclusive & Positive \\
\hline Pasta with pesto 2 & July 28 & Positive & Positive & Inconclusive & Positive \\
\hline Cooked chicken & July 28 & Negative & Negative & Negative & Negative \\
\hline Pesto jar 1 (Ohio) & July 28 & Negative & Negative & Negative & Negative \\
\hline Pesto jar 2 (Ohio) & July 28 & Negative & Negative & Negative & Negative \\
\hline Pesto jar 3 (Colorado) & unknown & Negative & NP & Negative & Negative \\
\hline Pesto jar 4 (California) & August 5 & $N P$ & NP & Negative & NP \\
\hline
\end{tabular}

Abbreviations: $\mathrm{ELISA}=$ enzyme-linked immunosorbent assay; $\mathrm{NP}=$ test not performed; $\mathrm{PCR}=$ polymerase chain reaction.

* Test for botulinum toxin type $B$.

† Test for botulinum toxin genes.

$\S$ Test for botulinum toxin type B-producing Clostridium spp.

vegetables, and company A Pine Nut Basil Pesto on July 13 at approximately 8:30 p.m. The pesto was poured over the chicken and pasta from an unopened glass jar and consumed by both patients without further heating. On July 28, investigators collected leftovers from this shared meal and two unopened jars of company A pesto and sent them to ODHL for testing. The pesto jar from the July 13 shared meal had been discarded. Botulinum toxin type B was detected in leftovers of pasta and pesto by ELISA. A culture of these food samples yielded Clostridium spp. that produced botulinum toxin type B, and PCR detected DNA encoding for type B toxin (Table).

Patient A received the pesto from a family member who had purchased several jars in May 2014 at a farm stand in San Clemente, California. Health officials in California collected and analyzed an unopened jar of the pesto from this family member's house. It was found to have a $\mathrm{pH}$ of 5.3 and water activity* of 0.965 (parameters insufficient to prevent growth of C. botulinum). Several jars also had been sent to family members in Colorado; one jar was collected and tested negative for botulinum toxin-producing Clostridium spp. and botulinum toxin at ODHL. Seven persons in Colorado reported that they ate company A pesto on May 29, and no illnesses were reported.

On July 29, 2014, CDPH began an investigation and discovered multiple jarred food items, including the Pine Nut Basil Pesto, available for sale on company A's website and farm stand. Neither company A nor the pesto manufacturer had permits or registrations allowing them to legally manufacture or sell

\footnotetext{
* Water activity is the amount of moisture that is available for bacterial growth Water activity of $>0.85$, in the absence of other controls, can allow growth of C. botulinum in a shelf-stable food product.
}

canned food, including food in jars, in California. CDPH investigators identified a lack of knowledge of safety issues involved with jarring foods and inadequate acidification and pressurization practices. There were no records indicating that critical factors (e.g., $\mathrm{pH}$, time, and temperature) were monitored during production. Invoices showed at least 39 jars of pesto were produced in 2014. After discussing the link between the cases in Ohio and company A pesto, company A voluntarily recalled all jarred food products. On July 30, CDPH posted Internet and social media notices warning consumers not to eat company A's jarred foods.

\section{Discussion}

This is the first reported botulism outbreak linked to pesto in the United States and the first reported worldwide in $>15$ years. A 1997 report described two botulism cases in Italy caused by home-canned pesto, also contaminated with botulinum toxin type B (1). Similar to this outbreak, both patients in Italy delayed seeking medical care until $\geq 6$ days after exposure.

The clinical diagnosis of botulism can be difficult. In the outbreak described here, both patients sought medical care multiple times before receiving a diagnosis of botulism, and patient $A$ was hospitalized for nearly 2 weeks before a clinician made the epidemiologic link between patient $A$ and patient $B$. If not for this clinician, the diagnoses might never have been made. In a 1995 outbreak of type B botulism linked to commercial chopped garlic in oil, a food vehicle similar to pesto, 36 previously unrecognized cases of botulism were identified only after two sisters with neurologic symptoms were evaluated (2). 


\section{Summary}

What is already known about this topic?

Foodborne botulism is a rare disease typically caused by consumption of improperly prepared and processed foods, including low-acid canned vegetables. A single case of foodborne botulism constitutes a public health emergency, necessitating an urgent response to identify the source and prevent further consumption of the toxin-containing food.

What is added by this report?

This report describes the first U.S. outbreak of botulism linked to pesto. The outbreak involved two patients, both of whom initially were examined for throat pain. The diagnosis of botulism was not made until nearly 2 weeks after symptom onset when both patients were hospitalized in the same health care facility. The pesto was produced without proper registration and licensure and sold commercially in jars at a farm stand and online.

What are the implications for public health practice?

As the demand for locally made, ready-to-eat food increases, consumers and public health officials should be aware of the risk for botulism from improperly canned foods such as pesto sold in jars. Producers of canned foods for commercial use should ensure that they adhere to food safety regulations.

Clinicians should maintain a high index of suspicion for botulism when evaluating patients with clinically compatible signs and symptoms. Throat pain, although not a classic feature of botulism, has been previously reported, having been attributed to severe dry mouth and throat caused by autonomic dysfunction (3). The classical presentation of botulism involves symmetric cranial nerve palsies, typically involving ptosis, blurry vision, dysphagia, and dysarthria, which is sometimes followed by symmetric descending flaccid paralysis, usually in the absence of sensory symptoms (3). Clinicians who suspect botulism should immediately call their state health department's emergency 24-hour telephone number. State health departments should call the
CDC Emergency Operations Center (770-488-7100) to arrange for rapid clinical consultation, and release of botulism antitoxin if indicated (4).

Consumer demand for fresh, farm-to-table foods has increased substantially during the past 15 years; for example, the number of farmers' markets in the United States nearly tripled from 2,863 in 2000 to 8,476 in 2015 (5). Consumers at farm stands and markets should be aware of the risk from improperly canned foods, including those in jars, produced without licensure and oversight from regulatory bodies. High-risk foods include low-acid canned foods (e.g., beans and peas) (G).

\footnotetext{
${ }^{1}$ Cincinnati Health Department; ${ }^{2}$ Food and Drug Branch, California Department of Public Health; ${ }^{3}$ Division of Foodborne, Waterborne, and Environmental Diseases, CDC; ${ }^{4}$ Ohio Department of Health Laboratory.
}

Corresponding author: Patrick Burke, Patrick.Burke@cincinnati-oh.gov, 513-357-7391.

\section{Acknowledgments}

Ohio Department of Health Outbreak Response and Bioterrorism Investigation Team. Cincinnati Health Department Food Safety Program. Colorado Department of Public Health and Environment.

\section{References}

1. Chiorboli E, Fortina G, Bona G. Flaccid paralysis caused by botulinum toxin type B after pesto ingestion. Pediatr Infect Dis J 1997;16:725-6. http://dx.doi.org/10.1097/00006454-199707000-00026

2. St Louis ME, Peck SHS, Bowering D, et al. Botulism from chopped garlic: delayed recognition of a major outbreak. Ann Intern Med 1988;108:363-8. http://dx.doi.org/10.7326/0003-4819-108-3-363

3. Sobel J. Botulism. Clin Infect Dis 2005;41:1167-73. http://dx.doi. org/10.1086/444507

4. CDC. Notice to readers: new telephone number to report botulism cases and request antitoxin. MMWR Morb Mortal Wkly Rep 2003;52:774.

5. Economic Research Service. Number of U.S. farmers markets has nearly tripled over the last 15 years. Washington, DC: US Department of Agriculture, Economic Research Service; 2015. http://www.ers.usda.gov/ data-products/chart-gallery/detail.aspx?chartId=53464

6. Peck MW. Clostridium botulinum and the safety of minimally heated, chilled foods: an emerging issue? J Appl Microbiol 2006;101:556-70. http://dx.doi.org/10.1111/j.1365-2672.2006.02987.x 